Health \& Medicine | Snežana Jovičić

\section{Quality benchmarks identified for laboratory medicine mobile apps}

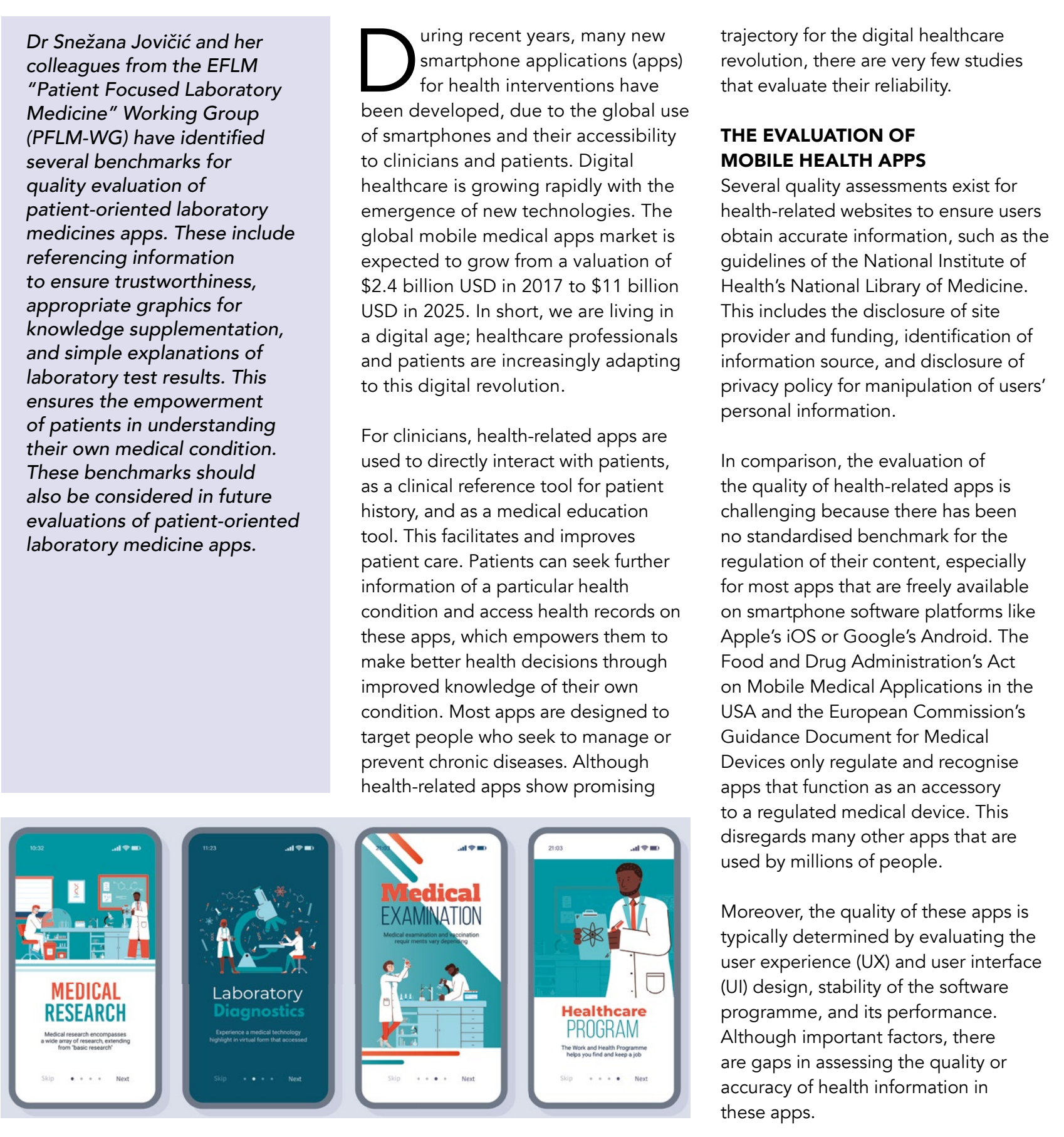

Many health-intervention apps include laboratory medicine data, laboratory test results. They are significant because they are needed to determine further clinical decisions. There is also a growing interest within patients to access and understand their laboratory test results on mobile apps, as it saves time and minimises the chances of patients missing key results. Allowing patients to access their laboratory results ensures a strong sense of ownership of their results, which increases patient engagement. Understanding of diseases allows patients to handle and monitor their health better over time. Therefore, the analysis of mobile health apps to be performed to ensure that both healthcare professionals and patients are receiving accurate health information from these mobile apps.

Dr Snežana Jovičić and her colleagues from the Working Group of the European Federation of Clinical Chemistry and Laboratory Medicine on Patient Focused Laboratory
Medicine (PFLM-WG) decided Medicine (PFLM-WG) decided to comprehensively evaluate the
quality and reliability of content and quality and reliability of content and functioning of health-related apps that utilise laboratory medicine data. A tool called the Mobile Application Rating Scale (MARS), which critiques the cover engagement, functionality, aesthetics, and information quality of the app health, wated aps with laborapry medicine data. MARS was devely in 2015 by Stoyanov et al and has been consistently used to evaluate healthrelated apps.

\section{RESULTS}

Apps that incorporate laboratory medicine data only represent a minority of health-related apps. Dr Jovičić grouped these apps into seven categories, which include apps that:

(1) provide medical advice about symptoms with the option of uploading laboratory test results for (2) inage and shaning,

(2) include reference ranges of cover

engagement

functionality

aesthetics

information

quality

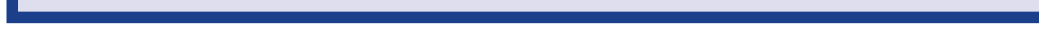

The researchers have used the Mobile Application Rating Scale (MARS) which criti
engagement, functionality, aesthetics, and information quality of the app content.

Although health-related apps show promising trajectory for the digital healthcare revolution, there are very few studies that evaluate their reliability.

information about the changes in test results, designed for patients, (3) provide quick reference of

laboratory test results for medical professionals,

(4) monitor patients' health using health parameters, such as glucose and cholesterol as laboratory data,
(5) provide patients' laboratory resuls to physicians,

(6) enable patients to access their laboratory test results directly from diagnostic centres, and

(7) provide electronic health records, including laboratory test results. It should be noted that less than half of the laboratory medicine data apps available are intended for patient designed for patients are of the poorest quality due to lack of quality which are generally not referenced. Moreover, there are significant privacy issues, as apps aimed for patient use generally have questionable affiliation of developers, which were mostly unknown or commercial. It is important to transparently disclose affiliated third parties, as personal laboratory medicin data can be collected, misused, and shared with parties that seek to exploit patients data without the explicit consent of users.

There may be a lack of correlation between ratings on app stores and a professional benchmark for critical 


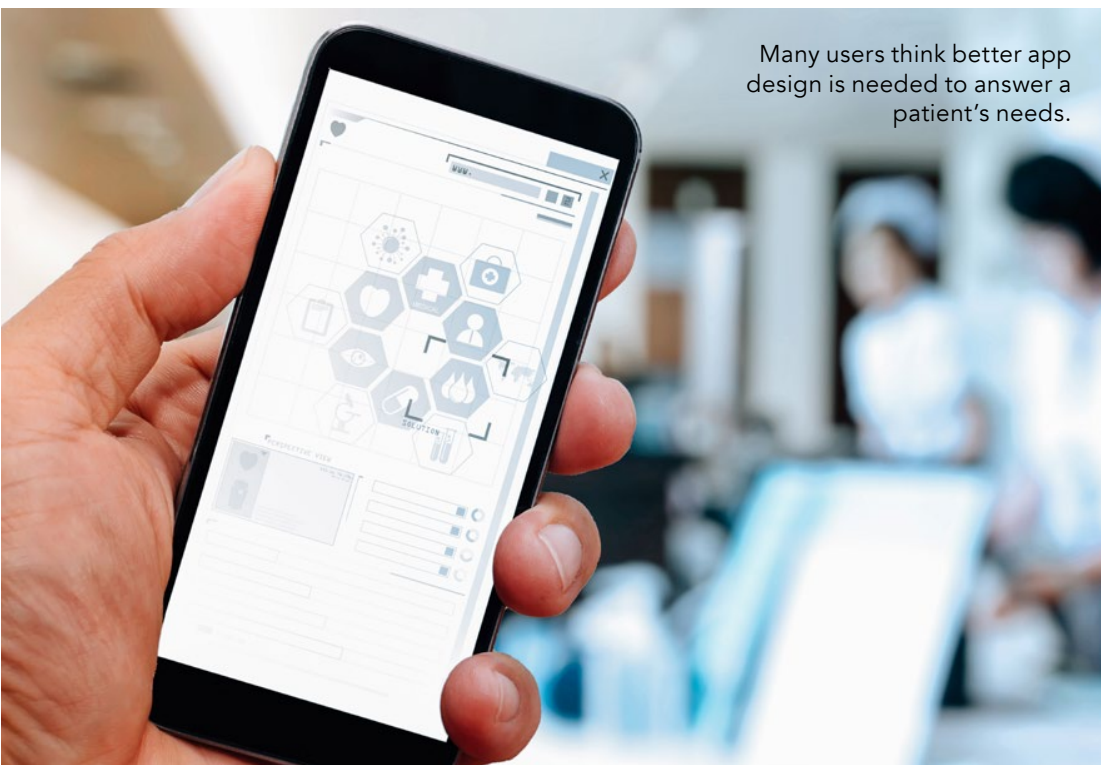

Information with visible references, appropriate graphics, and ease of usability must be considered by app developers to ensure patients can understand their own laboratory test results, and through them their medical condition.

evaluation. User ratings on app stores can be misleading as those writing them may not have enough medica knowledge to distinguish between useful and inaccurate information.

However, users' opinions are equally an alysis from the poine the gaps in potential patients. A further study was performed to gather the opinions and impressions of 25 potential patients on these apps. An adapted questionnaire of MARS was used to stimulate critical appraisal from participants. Participants' ge ranged from 20-60 years and were of both genders. This is then compared with the quality analysis of laboratory medicine specialists.

Participants corroborated the low utility of currently available laboratory medicine apps that were previously analysed by laboratory medicine specialists, signifying beter app design is needed to answer patients needs. They ident hed

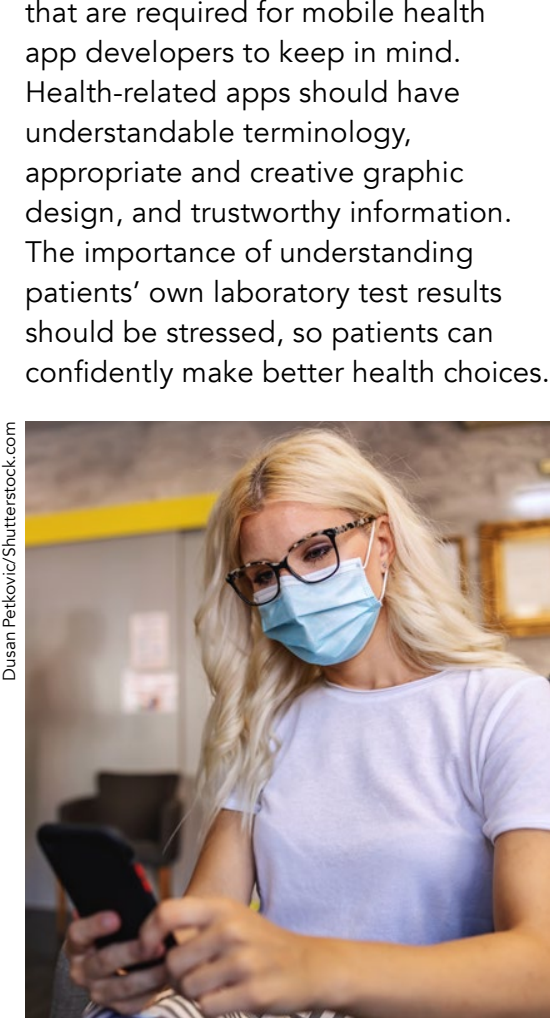

Patients are encouraged to fact-check informa
This requires the design of apps tha hearth paran the apps that are designed for patients include explanations of laboratory test results that are over-detailed with scientific terminology that is not explained, which may be more suited for healthcare professionals or patients who are more motivated to enquire further information. Therefore, simple explanations are needed to cater to patients with varying motivation and prior medical knowledge.

LIMITATIONS OF THE STUDY Even though the quality of healthrelated apps that include laboratory medicine data is influential, there lacks analysis on the effect of these apps health - did the app increase helth laboratory test results? The small number of participants were all healthy with no chronic conditions. There is a difference in motivation between healthy and sick individuals. Patients suffering from chronic diseases are more motivated to understand and search for more information about their health. Therefore, participants in these studies may negatively critique he apps more so than patients who may be more motivated to search for Regardless, this study has provided lamportant quality benchmark for aboratory qualine apps. Trustworthy visible references, appropriate graphics to supplement knowledge, and ease of usability must be considered by app developers to ensure patients can accurately understand their own laboratory test results, and through them their medical condition. In addition, these benchmarks should be considered when evaluating patientoriented laboratory medicine apps in future studies.

atients using laboratory medicine apps need to bear in mind the lack of regulation of the apps from government Therefore, acadenic institutions. Therefore, always fact-check and ensure visible references is utlised stated an

\section{(A) Behind the Research} Dr Snežana Jovičić

E: hionat@@gmail.com E: snezanaj@pharmacy.bg.ac.rs
W: https:/wwweflm eu/site/paga/a/1153

\section{Research Objectives}

Dr Jovičić and her colleagues have developed aboratory medicine apps.

\section{Detail}

Snežana Jovičić

Clinical Centre of Serbia

Centre for Medical Biochemistry

Department at the Clinic for Cardiac Surgery

Koste Todorovića 8, 11000 Belgrade

Serbia

Bio

Dr Snežana Jovičić is the Head of the Department at the Clinic for Cardiac Surgery of the Center for Medical Biochemistry, Clinical Center of Serbia, Teaching Assistant at the Department for Medical Biochemistry, Faculty of Pharmacy-University of Belgrade, and the Chair of the EFLM working group "Patient Focused

\section{Funding}

ration of Clinical Chemistry and Laboratory Medicine (EFLM)

\section{Collaborators}

Members of the EFLM "Patient Focused Laboratory (PFLM-WG): Ian D. Watson, oanna Siodmiak, Kristin Moberg Aakre, Wytze

Oosterhuis, Marta Duque Alcorta, Maximillian Kittel, Per Jørgensen, Vladimir Palicka, Marge Kutt, Mikko Anttonen, Mileva Georgieva Velizarova, and Jania Marc

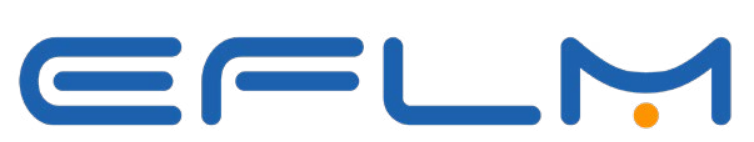

EUROPEAN FEDERATION OF CLINICAL CHEMISTRY AND LABORATORY MEDICINE

\section{References}

Jovičí, S. (2019). Smartphone applications for laboratory medicine - are they ready-to-use? [online]. AACC. Available at: $h$ https://www.aacc.org/science-and-research scientific-shorts/2019/smartphone-applications-tor laboratory-medicine [Accessed 07 January 2021].

Jovičić, S., Siodmiak, J. \& Watson, I. D. (2019). Quality evaluation of smartphone applications for laboratory (3), 388-397.

Jovičić, S., Siodmiak, J. Alcorta, M. D., Kittel, M. et al. (2020). Quality benchmarking of smartphone laboratory medicine applications: comparison of laboratory medicine specialists' and non-laboratory medicine professionals' evaluation. Clinical Chemistry and Laboratory Medici view/journals/cclm/ahead-of-print/article-10.1515 cclm-2020-0869/article-10.1515-cclm-2020-0869.xm [Accessed 07 January 2021].

Statista. (2018). Mobile medical apps market size worldwide in 2017, and a forecast for 2025 (in billion U.S dollars) [online]. 377 tista. Avaliable at: https://www.statista com/statistics/A77758/globalmobile-medical-appsmet-size/ [Accessed 07 January 2021].

\section{Personal Response}

How will you address the effects of laboratory medicine apps on patient behaviour in further studies? II In further studies, the WG intends to collaborate with patients' organisations on evaluating the effects
of laboratory medicine apps on behaviour of chronic patients, and on the outcome and management of their conditions. The planned finalisation of this project is to design a non-commercial laboratory medicine mobile that would be in accordance with the quality demands established through this study. If you want to learn more about the activities of the PFLM-WG, you may visit their webpage: https://www.eflm.eu/site/page/a/1153. II 